\title{
Big Five Inventory en Universitarios Peruanos: Resultados Preliminares de su Validación
}

\section{Big Five Inventory in Peruvian College Students: Preliminary Results of Its Validation}

\author{
Sergio Dominguez-Lara y César Merino-Soto \\ Universidad de San Martín de Porres
}

\author{
Bruno Zamudio \\ Universidad Inca Garcilaso de la Vega
}

\author{
Claudia Guevara-Cordero \\ Universidad Tecnológica del Perú
}

\begin{abstract}
El presente estudio tuvo como objetivo examinar la estructura interna del Big Five Inventory (BFI) en una muestra no probabilística incidental de 332 estudiantes entre 16 y 48 años $(M=20,39)$ de una universidad privada de Lima Metropolitana, Perú (82,83\% mujeres). Se realizó un análisis factorial exploratorio mediante el método de mínimos cuadrados no ponderados y rotación procrusteana oblicua con una matriz completamente especificada. También se calcularon las correlaciones lineales de Pearson entre los factores. La estructura factorial obtenida del BFI fue semejante a la estructura de la versión original de 44 ítems, aunque 7 ítems fueron eliminados. La versión de 37 ítems mostró buenos índices de consistencia interna, evaluada por alfa ordinal, omega y $H$. En conclusión, el BFI presenta indicadores psicométricos favorables que posibilitarían su uso en evaluaciones de grupo e investigación básica.
\end{abstract}

Palabras clave: cinco grandes factores, BFI, rotación procrusteana, estudiantes universitarios, validez, consistencia interna

\begin{abstract}
The aim of this study was to examine the internal structure of the Big Five Inventory (BFI) in a nonprobability sample of 332 students $(82.83 \%$ women) aged between 16 and 48 years $(M=20,39)$ who attended a private university in Metropolitan Lima, Peru. An exploratory factor analysis was performed using unweighted least squares and oblique Procrustes rotation with fully specified target matrix. In addition, the linear Pearson correlations between the factors were calculated. The BFI was found to have a factor structure similar to that of the original test, although 7 items were removed. The 37-items version displayed good internal consistency according to ordinal alpha, omega, and $H$. In conclusion, the BFI exhibits favorable psychometric indicators that suggest that it can be used in group evaluations and basic research.
\end{abstract}

Keywords: big five, BFI, Procrustes rotation, college students, validity, internal consistency

\section{Modelo de los Cinco Grandes Factores}

Desde que fue propuesto como una explicación organizada y parsimoniosa de la personalidad, el modelo de los cinco grandes factores (5GF) es quizá el más usado a nivel mundial para el estudio científico de la personalidad (Goldberg, 1990). Esto se respalda con los trabajos que corroboran su carácter intercultural a nivel sustantivo (McCrae \& Terracciano, 2005; Schmitt, Allik, McCrae \& Benet-Martínez, 2007; Yamagata et al., 2006) e, incluso, en relación a estilos de respuesta, como el aquiescente (Rammstedt, Kemper \& Borg, 2013). Sin embargo, aunque los resultados de algunos estudios sean divergentes a la premisa de replicabilidad intercultural (Fetvadjiev \& van der Vijver, 2015; Gurven, von Rueden, Massenkoff, Kaplan \& Lero Vie, 2013; Schmitt et al., 2007), se concluye que la replicación del modelo de los 5GF parece ser más

Sergio Dominguez-Lara, Escuela Profesional de Psicología e Instituto de Investigación de Psicología, Universidad de San Martín de Porres, Lima, Perú; César Merino-Soto, Escuela Profesional de Psicología e Instituto de Investigación de Psicología, Universidad de San Martín de Porres, Lima, Perú, y Centro de Investigación Transdisciplinar de Psicología, Universidad Autónoma del Estado de Morelos, Cuernavaca, Perú; Bruno Zamudio, Facultad de Psicología y Trabajo Social, Universidad Inca Garcilaso de la Vega e Instituto de Promoción de la Mujer, Infancia y Familia, Lima, Perú; Claudia Guevara-Cordero „Psicología, Universidad Tecnológica del Perú y Facultad de Psicología, Universidad Nacional Mayor de San Marcos, Lima, Perú.

La correspondencia relativa a este artículo debe ser dirigida a Sergio Dominguez-Lara, Instituto de Investigación de Psicología, Universidad de San Martín de Porres, Avda. Tomás Marsano 242 (5 piso), Lima 34, Perú. E-mails: sdominguezl@usmp.pe, sdominguezmpcs@gmail.com 
robusta en sociedades occidentales, con educación formal, industrializadas, pudientes y democráticas (Henrich, Heine \& Norenzayan, 2010), así como en población universitaria o con grado de instrucción elevado (Rammstedt, Kemper \& Borg, 2013) y con edades cercanas a la adultez (Soto, John, Gosling \& Potter, 2008), al ser menos susceptibles a mostrar sesgos de respuesta.

El estudio de los 5GF y su relación con diversas variables psicológicas tiene amplia bibliografía, cuya cobertura de investigación es internacional, y, si bien al principio no podía considerarse al modelo de los 5GF como efectivamente predictivo, la covariación observada entre las dimensiones de los 5GF y el desempeño conductual en muchas áreas de comportamiento humano ha sido de magnitud elevada o moderada, incluso con conductas disfuncionales. Esto respalda la consistencia teórica e incluso práctica, apoyando el argumento de ser un modelo no solo que puede ser de valor teórico, sino también aplicativo. Esta es una de las razones por la que actualmente los 5GF complementan conceptualmente a uno de los manuales diagnósticos más usados en el mundo: el DSM-5 (Krueger et al., 2011), pues la replicabilidad internacional de sus hallazgos lo hace un modelo fiable.

\section{Medición de los Cinco Grandes}

El presente trabajo ponderó el contenido metodológico, porque es el aspecto menos abordado en relación al estudio instrumental del Big Five Inventory (BFI), en desmedro del contenido sustantivo. Dado que este problema es más notorio en Hispanoamérica, se orientó el contenido a este aspecto inusualmente desarrollado. De este modo, en cuanto a las propuestas de su medición, se han diseñado medidas basadas principalmente en el autoinforme y en el heteroinforme. Este último recoge la descripción del sujeto desde un formato de cuestionario preparado para ser respondido por una persona cercana a él. En cuanto a los autoinformes, existen diversos instrumentos para evaluar los 5GF, pero dentro de aquellos considerados breves y de mayor uso destacan dos: el NEO Five-Factor Inventory (NEO FFI; Costa \& McCrae, 1992), de 60 ítems, y el BFI (John, Donahue \& Kentle, 1991), de 44 reactivos. Cada uno de ellos cuenta con una cantidad elevada de investigaciones instrumentales, encontrándose propiedades psicométricas apropiadas de ambos en diversos países. No obstante, una de las ventajas del BFI es que se distribuye libremente, mientras que el NEO-FFI es un test comercial, lo que implica que el estudio de sus propiedades psicométricas y modificación/refinamiento de los ítems no sería posible (Goldberg et al., 2006). En este sentido, el BFI parece también una opción viable por otros motivos: (a) cuenta con una versión adaptada para su uso en países de habla hispana, pero, a diferencia del NEO-FII, lo hace en un español estándar (Benet-Martínez \& John, 1998), es decir, se adecúa a su lectura internacional y está poco influenciado por el fraseo local de cada lugar y no solo se orienta para determinados países; (b) es breve, en comparación con los 240 ítems del Inventario de Personalidad NEO Revisado (NEO-PI-R) y 60 del NEO-FII; (c) muestra buenas propiedades psicométricas en estudios previos; (d) presenta similitudes con las facetas del NEO-PI-R, es decir, los ítems del BFI se distribuyen las facetas del NEO-PI-R y muestrean su contenido de una manera que puede representarlo eficientemente (Soto \& John, 2009) y (e) posibilita la extracción de versiones más cortas (e.g., Rammstedt \& John, 2007). Aun con esas ventajas, sorprende que no existan estudios factoriales publicados del BFI en participantes adultos peruanos o sudamericanos. Cabe precisar que, si bien existe una traducción al español hablado en Argentina y orientado a adolescentes de ese país (Castro Solano \& Casullo, 2001), sus indicadores psicométricos no son favorables: bajas confiabilidades de las puntuaciones y elevada complejidad factorial, esto es, que la variación psicométrica de un ítem es explicada por su propio factor y por factores no destinados a medirlo (Merino \& Grimaldo Muchotrigo, 2011).

\section{Estrategias Analítico-Factoriales Asociadas al Estudio de los Cinco Grandes}

Respecto de las estrategias de verificación de la estructura factorial del BFI, existe una diversidad de procedimientos analíticos factoriales utilizados. Aunque se cuenta con procedimientos disponibles de análisis exploratorios enfocados en los factores comunes (e.g., ejes principales, mínimos cuadrados no ponderados), en la mayoría de los estudios se aplica el enfoque conocido como Little Jiffy, que se compone de tres procedimientos: el uso de regla de Kaiser (retener componentes con autovalores mayores que la unidad), el análisis de componentes principales y la rotación Varimax (Ferrando \& Anguiano-Carrasco, 2010). Esto se observa en los estudios de las versiones en español (Benet-Martínez \& John, 1998; Reyes Zamorano, Álvarez Carrillo, Peredo Silva, Miranda Sandoval \& Rebolledo Pastrana, 2014), holandés (Denissen, Geenen, van Aken, Gosling \& Potter, 2008), italiano (Fossati, Borroni, Marchione \& Maffei, 2011; Ubbiali, Chiorri, Hampton \& Donati, 2013), tsimane (Gurven et al., 2013) y chino (Carciofo, Yang, Song, Du \& Zhang, 2016). 
No obstante, se sabe que los análisis exploratorios no se guían por la teoría y no se controlan los aspectos que permiten hacer una verificación del cumplimiento de los parámetros, como los errores correlacionados y la configuración de los ítems (Aluja \& Blanch, 2003). Además, el Little Jiffy está ampliamente desestimado por la comunidad científica (e.g., Ferrando \& Anguiano-Carrasco, 2010; Lloret-Segura, Ferreres-Traver, Hernández-Baeza \& Tomás-Marco, 2014), debido a que tienden a sobreestimar la cantidad de factores a extraer y los coeficientes de configuración (cargas factoriales) resultan espuriamente altos (Dominguez Lara, 2014), lo que podría dar la impresión de que los ítems se identifican de forma casi perfecta con el factor correspondiente. En su lugar, sería más apropiado utilizar procedimientos de análisis factorial propiamente dicho (e.g., mínimos cuadrados no ponderados; ver Worrell \& Cross Jr., 2004), los cuales analizan la estructura factorial en función de la varianza compartida entre los ítems.

Además, es necesario resaltar que la rotación Varimax fuerza la ortogonalidad de los factores, pero existe evidencia acerca de la relación entre las dimensiones del modelo de los 5GF. Quizá este es el panorama observado en la mayoría de estudios citados que usaron el análisis de componentes principales, es decir, probablemente la cantidad de varianza explicada no se refiera únicamente a la varianza común, como es lo esperado, sino que esté contaminada con otro tipo de variabilidad (e.g., especificidad).

En cuanto al uso del análisis factorial confirmatorio (AFC) en los instrumentos basados en el modelo de los 5GF, de forma consistente se han obtenido pobres niveles de ajuste (e.g., Gurven et al., 2013; Leung, Wong, Chan \& Lam, 2013). Esto se debe a que el AFC parte del supuesto de que cada ítem es una medida factorialmente simple de un rasgo, es decir, que no es afectado por los demás factores. Ello es una suposición poco realista en los modelos de personalidad (Ferrando \& Lorenzo-Seva, 2000), más aún el de los 5GF (McCrae, Zonderman, Costa Jr., Bond \& Paunonen, 1996), porque existe evidencia de que los ítems tienden a la complejidad factorial (Ferrando \& Lorenzo-Seva, 2014) y solo en ocasiones se acercan a una estructura factorialmente simple (Kaiser, 1974). Asimismo, no hay razones conceptuales que indiquen que las cargas factoriales en los factores secundarios sean cero en el modelo de los 5GF (McCrae et al., 1996), por lo que es necesario un procedimiento más de acuerdo con las bases conceptuales de un modelo que admita varianza compartida entre varios de sus factores (Aluja, García, García \& Seisdedos, 2005; García, Escorial, García, Blanch \& Aluja, 2012; Yang, 2010). Finalmente, si se opta por un AFC y se conoce que los constructos estudiados inherentemente poseen algún grado de complejidad factorial, se estaría cometiendo un error de especificación. En síntesis, existe una mala especificación cuando uno o más parámetros son especificados, aun cuando sus valores poblacionales son cero o sobre-parametrización o cuando algunos parámetros son fijados a cero, pero sus valores poblacionales son diferentes de cero, lo que se conoce como infraparametrización (Hu \& Bentler, 1998). En ambos casos derivaría en un modelo inadecuado desde el principio y los índices de ajuste se verían afectados negativamente, concluyendo, así, que el modelo no se ajusta a los datos, cuando, en realidad, el diseño analítico no se corresponde con la naturaleza de los datos.

\section{Rotación Procrusteana y su Uso en el Estudio de los Cinco Grandes}

Una aproximación menos restrictiva que el AFC es la rotación procrusteana, que parte de un principio sencillo: evaluar el grado de ajuste de una matriz de cargas factoriales hacia una matriz objetivo o target matrix (TM; McCrae et al., 1996) especificada previamente; por lo tanto, se fuerzan los datos lo máximo posible, a fin de aproximarse a la estructura planteada en la TM (Digman, 1967). Este procedimiento es una forma de rotación que es guiada por una especificación previa de las relaciones entre los ítems y los factores, y, por lo tanto, facilita el contraste de hipótesis sobre la configuración de los ítems.

Existen diversos grados de especificación de la TM; por ejemplo, en la rotación procrusteana completamente especificada cada uno de los ítems presenta idealmente un coeficiente de configuración de magnitud uno en solo un factor y cero en los demás factores. De ese modo, un ajuste adecuado brindaría coeficientes de configuración elevados en un factor para cada uno de los ítems y cercanos a cero en los factores secundarios. No obstante, en estos métodos puede especificarse la magnitud de los coeficientes de configuración, planteando de antemano la presencia de baja o moderada complejidad factorial de los ítems, dada las características del modelo, lo que resulta apropiado para instrumentos derivados del modelo de los 5GF. Respecto de este punto, en algunos estudios el factor Afabilidad muestra correlaciones inversas con Neuroticismo (Benet-Martínez \& John, 1998; Denissen et al., 2008; Gurven et al., 2013) y directas con Apertura (Gurven et al., 2013) y Responsabilidad (Denissen et al., 2008; Gurven et al., 2013), mientras que Extraversión se relaciona directamente con Apertura (Benet-Martínez \& John, 1998; Denissen et al., 2008; García et al., 2012; Gurven et al., 2013), Afabilidad (Denissen et al., 2008; García et al., 2012; Gurven et al., 
2013) y Responsabilidad (Denissen et al., 2008; Gurven et al., 2013). Por su parte, Neuroticismo se asocia de forma inversa con Extraversión (Benet-Martínez \& John, 1998; Denissen et al., 2008; García et al., 2012; Gurven et al., 2013), Apertura (Gurven et al., 2013) y Responsabilidad (Benet-Martínez \& John, 1998; Denissen et al., 2008; García et al., 2012; Gurven et al., 2013) y este último se relaciona directamente con Apertura (Gurven et al., 2013). En tal sentido, sería esperable que en la configuración final del instrumento esta situación se vea reflejada en los índices de simplicidad factorial y en las correlaciones interfactoriales.

Con relación a este tipo particular de rotación, destacan diversas propuestas de rotaciones procrusteanas, tanto ortogonales (Browne, 1972a; Cliff, 1966; Schönemann, 1966) como oblicuas (Browne, 1972b), siendo más conservadora la ortogonal (McCrae et al., 1996), aunque sin perder de vista las relaciones esperadas entre constructos analizados.

Por lo expuesto, el objetivo de este estudio fue examinar la estructura interna desde una perspectiva menos restrictiva que la del AFC (Ferrando \& Lorenzo-Seva, 2000), utilizando una rotación procrusteana completamente especificada oblicua. De este modo, se especificaron cargas factoriales cercanas a la unidad en su factor principal y diferentes de cero en los factores secundarios, ya que se esperaba algún grado de complejidad factorial. Asimismo, se permitió la correlación entre factores (rotación oblicua), debido a la evidencia presentada anteriormente que da cuenta de que existe relación entre los factores del modelo de los 5GF. Respecto de la asociación entre factores, y en vista de la evidencia mostrada, se esperaba una relación directa entre Extraversión y Afabilidad y Apertura y una relación inversa de Neuroticismo y Extraversión, Afabilidad y Responsabilidad. Finalmente, se realizó un reporte de confiabilidad con respecto a la consistencia interna de las puntuaciones observadas y variables latentes.

\section{Método}

\section{Participantes}

Fueron evaluados 332 estudiantes de psicología de $1^{\text {er }}$ al $7^{\circ}$ ciclo pertenecientes a una universidad privada ubicada en Lima Metropolitana, Perú (82,83\% de mujeres; una persona no consignó ese dato), institución en la que, de manera estimativa, predominantemente se matriculan estudiantes de nivel socioeconómico (NSE) medio y en menor proporción medio-bajo y medio-alto. La edad estuvo comprendida entre 16 y 48 años $(M=20,39, D E=3,97)$ y fue ligeramente mayor en varones, $t(325)=2,242, p=0,026$, $d=0,249,95 \% \mathrm{IC}_{\text {diferencia }}[0,158,2,421]$.

El muestreo utilizado fue no probabilístico incidental; sin embargo, se procuró que la muestra tuviera una proporción similar del NSE de la población de origen. Todos los participantes mostraron disponibilidad y ninguno rechazó su inclusión en el estudio.

\section{Instrumento}

Big Five Inventory (John et al., 1991). Es una medida de autoinforme que, mediante 44 ítems, evalúa los 5GF: Extraversión (8 ítems; ...es extrovertido, sociable), Afabilidad (9 ítems; ...es generoso), Responsabilidad (9 ítems; ...persevera hasta terminar el trabajo), Neuroticismo (8 ítems; ...con frecuencia se pone tenso) y Apertura (10 ítems; ...valora lo artístico y lo estético). Sus puntuaciones se obtienen por la suma simple de los ítems: a mayor puntuación mayor presencia del atributo en el evaluado. Cada ítem está escalado en un formato ordinal de cinco puntos que va desde 1 Muy en desacuerdo hasta 5 Muy de acuerdo y en las instrucciones se solicita al evaluado indicar el grado de acuerdo con cada uno de los ítems. Fue utilizada la versión española de Benet-Martínez y John (1998), la cual tuvo como base la traducción del BFI-44 (John et al., 1991), que fue realizada en idioma inglés. Esa versión en español del BFI tuvo evidencias favorables con respecto a su estructura interna de cinco dimensiones, intercorrelación significativa entre estas, así como indicadores de confiabilidad favorables ( $a_{\text {promedio }}=0,78$; Benet-Martínez \& John, 1998).

Antes de la aplicación del material, los ítems fueron muestreados a un grupo pequeño $(n=10)$ de participantes para verificar su claridad. Ninguno de ellos expresó problemas para comprender el material ni para dar respuesta al formato del instrumento. 


\section{Procedimiento}

Para desarrollar el estudio, se solicitó a las autoridades universitarias el permiso para realizar las evaluaciones en el horario habitual de clases. Asimismo, el Instituto de Investigación de Psicología de la institución dio el aval correspondiente sobre los aspectos éticos y procedimentales de la investigación. Las aplicaciones fueron realizadas previa coordinación con los docentes. Los participantes fueron informados sobre los objetivos de la investigación y firmaron un formulario de consentimiento informado. La participación de cada estudiante fue voluntaria y con la opción de abandonar la evaluación si no deseaba seguir. Además, no fue recompensada académica ni económicamente. El instrumento fue aplicado de forma colectiva en horarios de clase por dos de los investigadores, quienes brindaron instrucciones estandarizadas, durando la aplicación del BFI un promedio de 25 minutos. Se monitoreó su aplicación y ninguno de los participantes requirió explícitamente aclaraciones sobre el contenido de los ítems.

\section{Análisis de Datos}

Antes de llevar a cabo los análisis, fue revertida la puntuación de los ítems que evalúan de forma inversa el constructo. Luego se efectuó un análisis de los supuestos estadísticos previos al análisis factorial.

Estructura factorial del BFI. A fin de examinar la estructura factorial del BFI, se realizó un análisis factorial exploratorio (AFE) por mínimos cuadrados no ponderados, aplicando una rotación procrusteana completamente especificada y oblicua (Browne, 1972b), con una matriz de correlaciones policóricas inter-ítem (Dominguez Lara, 2014). Para la determinación del número de factores se utilizó el análisis paralelo (Horn, 1965), usando una matriz de variables aleatorias y también correlaciones policóricas entre ellas. El uso de este tipo de correlaciones permite representar la asociación entre variables continuas latentes, pero empíricamente expresadas en variables ordinales. Para realizar estos análisis fue usado el programa FACTOR (Lorenzo-Seva \& Ferrando, 2013).

Luego de ello, se configuraron tres TM con especificaciones diferentes para realizar la rotación de la matriz empírica de los ítems hacia ellas. En la primera $\left(\mathrm{TM}_{1}\right)$, a cada ítem se le asignó un coeficiente de configuración igual a la unidad en el factor teóricamente correspondiente y 0,15 en los otros factores. Esta especificación declaraba que el ítem contenía la máxima varianza con su factor correspondiente, mientras mantenía relaciones triviales no-cero con el resto de factores. La carga planteada para la $\mathrm{TM}_{1}$ representó un balance entre un criterio estricto y habitual en el AFC (cargas cero) y el límite que a menudo se usa para identificar cargas aceptables en un factor $(0,30)$. Para las $\mathrm{TM}_{2}$ y $\mathrm{TM}_{3}$ fue relajándose progresivamente dicha exigencia hasta un nivel aún considerado elevado $(0,80)$. Así, en la segunda $\left(\mathrm{TM}_{2}\right)$ se estableció que cada ítem mantenía un coeficiente de configuración de 0,90 en su factor y 0,15 en los otros factores. Esta especificación relajó levemente la fuerza de la relación ítem-factor. En la última $\left(\mathrm{TM}_{3}\right)$ los ítems fueron fijados con coeficientes de configuración igual a 0,80 en su factor correspondiente y 0,15 en los otros factores. La especificación se planteó de ese modo, ya que, como se mencionó en la sección introductoria, teóricamente se esperaba una correlación entre los factores, al margen de que se trate de un AFE o AFC, por lo cual es poco realista fijar en cero las cargas factoriales de cada ítem en los factores secundarios, al momento de especificar la matriz (ten Berge, 1986).

En contraste con el AFC, que requiere de índice de ajuste del modelo a los datos, el enfoque utilizado en el presente estudio usa principalmente la similitud cuantitativa de la matriz-objetivo y la matriz empírica rotada, por medio del coeficiente de congruencia factorial ( $\Phi$; Lorenzo-Seva \& ten Berge, 2006; Tucker, 1951), el cual cuantifica el grado de similitud entre la TM y la matriz resultante. Un coeficiente $\Phi \geq 0,85$ es considerado adecuado (ten Berge, 1986) y $\Phi \geq 0,95$ indicaría la replicabilidad, tanto a nivel de factor como de ítem (Lorenzo-Seva \& ten Berge, 2006; McCrae et al., 1996; Ortet et al., 2007), es decir, que la TM especificada se replica satisfactoriamente con los datos de la muestra.

Junto con el análisis de la congruencia factorial, fueron implementados dos procedimientos para extender la evaluación de las evidencias internas de validez convergente. Estos consistieron en evaluar las correlaciones entre los factores, esperando que fueran teóricamente relevantes y estadísticamente significativas, y evaluar la validez discriminante de los ítems, mediante el cálculo del índice de simplicidad factorial (ISF; Fleming \& Merino Soto, 2005; Kaiser, 1974) para los ítems y para el vector de ítems de cada factor. El ISF evalúa el grado de diferenciación empírica de los ítems de un factor con respecto a los demás factores. Según estándares exigentes, un ISF $<0,80$ puede considerarse como factorialmente complejo, es decir, los ítems serían explicados por más de un constructo simultáneamente (Merino \& Grimaldo 
Muchotrigo, 2011). Sin embargo, aunque normalmente la complejidad factorial se considera como un indicador desfavorable, en este estudio se aplicó un criterio liberal para interpretarla, es decir, se consideraron otros indicadores adicionales (e.g., $\Phi)$, ya que no puede esperarse que los ISF sean elevados - considerando las características de los $5 \mathrm{GF}$ - y la especificación de que las cargas secundarias pudieran ser no-cero. De este modo, se maximizaría la incorporación de ítems en la versión final del instrumento.

Relación entre factores. En vista de las hipótesis relacionales planteadas en la sección introductoria, el grado de asociación entre factores fue evaluado por medio del coeficiente de correlación de Pearson, considerándolo como significativo si su magnitud era mayor que 0,20 (Ferguson, 2009).

Confiabilidad por consistencia interna. Se utilizó el coeficiente alfa de Cronbach y sus intervalos de confianza (IC; Dominguez-Lara \& Merino-Soto, 2015) con el método de Fisher (1950). Este método es el que brinda mejores estimaciones (Romano, Kromrey \& Hibbard, 2010; Romano, Kromrey, Owens \& Scott, 2011), esperando magnitudes $\geq 0,70$ (Merino Soto, Navarro Loli \& García Ramirez, 2014). Del mismo modo, se calculó alfa ordinal (Dominguez-Lara, 2012; Elosua Oliden \& Zumbo, 2008), el coeficiente Omega ( $\omega$; McDonald, 1999) y el coeficiente $H$ para conocer la confiabilidad de los constructos (Dominguez-Lara, 2016; Hancock \& Mueller, 2001). Sobre la confiabilidad, cabe resaltar que si las condiciones para aplicar el coeficiente alfa no son cumplidas (tau-equivalencia), es conveniente optar por alternativas que no dependan de esa restricción y cuya estimación no se vea afectada por el número de ítems.

\section{Resultados}

\section{Análisis Estructural del BFI y Relación entre Factores}

La matriz de correlaciones presentó las condiciones adecuadas para ser factorizada: KMO = 0,842 y test de esfericidad de Bartlett significativo $(p<0,001)$. No se observó multicolinealidad entre los ítems y se constató la normalidad multivariada a través del coeficiente de Mardia (1970), con un indicador de 33,63, valor considerado apropiado para continuar con el procesamiento (<70; Rodríguez Ayán \& Ruiz Díaz, 2008). El análisis paralelo indicó que sería conveniente extraer cinco factores, los cuales explicaron de forma conjunta el 49,46\% de la variabilidad de las puntuaciones (Tabla 1).

Tabla 1

Análisis Paralelo del BFI

\begin{tabular}{cccc}
\hline Factor & $\begin{array}{c}\text { Autovalor } \\
\text { real }\end{array}$ & $\begin{array}{c}\text { Autovalor } \\
\text { aleatorio }\end{array}$ & $\begin{array}{c}\text { Varianza explicada } \\
\text { acumulada (\%) }\end{array}$ \\
\hline 1 & 8,267 & 1,696 & 22,343 \\
2 & 3,420 & 1,613 & 31,587 \\
3 & 2,623 & 1,546 & 38,676 \\
4 & 2,009 & 1,490 & 44,107 \\
5 & 1,978 & 1,442 & 49,457 \\
6 & 1,281 & 1,397 & - \\
\hline
\end{tabular}

Al evaluar las tres TM (1,00 y 0,15; 0,90 y 0,$15 ; 0,80$ y 0,15$)$, los datos se ajustan mejor a la $\mathrm{TM}_{2}$ (cargas factoriales de 0,90 en el factor principal y 0,15 en los factores secundarios), obteniéndose coeficientes de congruencia de mayor magnitud, tanto a nivel de ítems como de factor, y menor cantidad de ítems eliminados por excesiva complejidad factorial (Tabla 2). De forma sucesiva fueron eliminados siete ítems: ítem 2 ("Me veo a mí mismo/a como alguien que: Tiende a ser criticón"), 13 ("Inicia disputas con los demás"), 22 ("Es a veces maleducado con los demás"), 28 ("Es indulgente, no le cuesta perdonar") y 33 ("Es a veces frío y distante") de Afabilidad; ítem 8 (“A veces puede ser algo descuidado") de Responsabilidad y el ítem 36 ("Le gusta reflexionar, jugar con las ideas") de Apertura; todos ellos por excesiva complejidad factorial.

Los ISF a nivel de ítem evidencian poca diferenciación empírica, pero los $\Phi$ resultan adecuados para la mayoría de los ítems: Extraversión $\left(\mathrm{ISF}_{\text {promedio }}=0,574 ; \Phi_{\text {promedio }}=0,854\right)$, Afabilidad $\left(\mathrm{ISF}_{\text {promedio }}=0,583\right.$; $\left.\Phi_{\text {promedio }}=0,901\right)$, Responsabilidad $\left(\operatorname{ISF}_{\text {promedio }}=0,554 ; \Phi_{\text {promedio }}=0,844\right)$, Neuroticismo $\left(\operatorname{ISF}_{\text {promedio }}=0,732 ;\right.$ 
$\left.\Phi_{\text {promedio }}=0,918\right)$ y Apertura $\left(\mathrm{ISF}_{\text {promedio }}=0,573 ; \Phi_{\text {promedio }}=0,882\right)$. La congruencia total entre la matriz factorial derivada de los datos y la TM fue de 0,863, valor considerado adecuado (Lorenzo-Seva \& ten Berge, 2006). Por último, con respecto a la relación entre puntuaciones directas de cada uno de los factores, se observan correlaciones significativas (> 0,20; Tabla 3), aunque el patrón relacional de esos factores luego de la rotación procrusteana (Tabla 2) presenta diferencias, razones que serán discutidas más adelante.

Tabla 2

Análisis Descriptivo, Estructural y de Confiabilidad del BFI

\begin{tabular}{|c|c|c|c|c|c|c|c|c|c|c|c|}
\hline & $M$ & $D E$ & $g_{1}$ & $g_{2}$ & $\mathrm{~F}_{1}$ & $\mathrm{~F}_{2}$ & $\mathrm{~F}_{3}$ & $\mathrm{~F}_{4}$ & $\mathrm{~F}_{5}$ & $\Phi_{\text {I }}$ & ISF \\
\hline BFI1 & 3,63 & 1,045 & $-0,563$ & $-0,217$ & 0,786 & 0,440 & 0,061 & 0,290 & 0,254 & 0,928 & 0,574 \\
\hline BFI6 (I) & 2,36 & 1,092 & 0,701 & $-0,117$ & 0,438 & $-0,090$ & $-0,231$ & 0,049 & $-0,078$ & 0,704 & 0,689 \\
\hline BFI11 & 3,84 & 0,948 & $-0,656$ & 0,068 & 0,574 & 0,500 & 0,245 & 0,075 & 0,118 & 0,853 & 0,503 \\
\hline BFI16 (I) & 3,29 & 1,192 & $-0,122$ & $-1,048$ & 0,835 & 0,054 & 0,185 & 0,139 & 0,196 & 0,992 & 0,851 \\
\hline BFI27 (I) & 3,00 & 1,132 & 0,113 & $-0,950$ & 0,670 & $-0,239$ & 0,152 & $-0,013$ & 0,348 & 0,837 & 0,632 \\
\hline BFI32 & 3,66 & 0,962 & $-0,469$ & $-0,205$ & 0,525 & 0,504 & 0,265 & 0,207 & 0,244 & 0,824 & 0,364 \\
\hline BFI40 & 3,70 & 1,048 & $-0,638$ & $-0,223$ & 0,388 & 0,290 & 0,106 & $-0,025$ & 0,362 & 0,788 & 0,403 \\
\hline BFI43 & 3,71 & 1,084 & $-0,573$ & $-0,441$ & 0,810 & 0,538 & 0,025 & 0,204 & 0,267 & 0,906 & 0,575 \\
\hline BFI7 & 4,20 & 0,808 & $-1,116$ & 1,894 & 0,364 & 0,584 & 0,265 & 0,120 & 0,152 & 0,913 & 0,516 \\
\hline BFI24 & 3,60 & 1,071 & $-0,617$ & $-0,205$ & 0,239 & 0,431 & $-0,097$ & 0,166 & 0,089 & 0,879 & 0,578 \\
\hline BFI37 & 4,06 & 0,844 & $-1,079$ & 1,583 & 0,167 & 0,583 & 0,135 & $-0,094$ & $-0,032$ & 0,923 & 0,825 \\
\hline BFI41 & 4,11 & 0,855 & $-1,259$ & 2,248 & 0,367 & 0,543 & 0,265 & 0,134 & 0,254 & 0,887 & 0,415 \\
\hline BFI3 & 3,66 & 1,044 & $-0,574$ & $-0,229$ & 0,163 & 0,198 & 0,728 & 0,495 & 0,523 & 0,861 & 0,413 \\
\hline BFI14 & 4,13 & 0,836 & $-0,965$ & 1,112 & 0,097 & 0,429 & 0,481 & 0,106 & 0,205 & 0,851 & 0,489 \\
\hline BFI18 (I) & 3,19 & 1,118 & $-0,021$ & $-0,844$ & 0,130 & $-0,093$ & 0,621 & 0,144 & 0,046 & 0,949 & 0,861 \\
\hline BFI21 & 4,00 & 0,921 & $-1,125$ & 1,568 & 0,190 & 0,279 & 0,681 & 0,230 & 0,514 & 0,886 & 0,459 \\
\hline BFI25 (I) & 3,53 & 1,075 & $-0,328$ & $-0,614$ & 0,064 & $-0,077$ & 0,615 & 0,045 & $-0,019$ & 0,937 & 0,960 \\
\hline BFI29 & 3,90 & 0,804 & $-0,802$ & 1,089 & 0,247 & 0,340 & 0,493 & 0,236 & 0,456 & 0,810 & 0,279 \\
\hline BFI34 & 3,55 & 0,913 & $-0,428$ & $-0,183$ & 0,133 & 0,399 & 0,473 & 0,258 & 0,159 & 0,853 & 0,408 \\
\hline BFI42 (I) & 2,72 & 1,134 & 0,248 & $-0,755$ & $-0,140$ & $-0,350$ & 0,560 & $-0,133$ & 0,079 & 0,606 & 0,566 \\
\hline BFI4 & 2,37 & 1,170 & 0,531 & $-0,660$ & $-0,033$ & 0,020 & 0,023 & 0,656 & 0,245 & 0,944 & 0,851 \\
\hline BFI9 (I) & 2,91 & 1,046 & 0,172 & $-0,626$ & 0,241 & $-0,027$ & 0,168 & 0,807 & 0,052 & 0,969 & 0,852 \\
\hline BFI15 & 2,96 & 1,067 & $-0,012$ & $-0,714$ & $-0,044$ & 0,204 & 0,083 & 0,635 & 0,058 & 0,962 & 0,857 \\
\hline BFI19 (I) & 2,88 & 1,062 & 0,212 & $-0,642$ & 0,100 & $-0,179$ & 0,198 & 0,673 & 0,121 & 0,913 & 0,783 \\
\hline BFI26 & 3,73 & 0,951 & $-0,705$ & 0,118 & 0,117 & 0,541 & 0,426 & 0,571 & 0,098 & 0,803 & 0,406 \\
\hline BFI30 & 3,06 & 1,163 & $-0,089$ & $-0,859$ & 0,167 & 0,181 & 0,085 & 0,754 & 0,224 & 0,989 & 0,787 \\
\hline BFI35 (I) & 2,37 & 0,944 & 0,592 & $-0,066$ & 0,210 & $-0,309$ & 0,254 & 0,794 & 0,226 & 0,864 & 0,642 \\
\hline BFI38 & 3,12 & 1,100 & $-0,015$ & $-0,675$ & 0,019 & 0,445 & 0,098 & 0,691 & 0,004 & 0,900 & 0,679 \\
\hline BFI5 & 3,97 & 0,891 & $-0,823$ & 0,727 & 0,355 & 0,258 & 0,517 & 0,381 & 0,688 & 0,859 & 0,335 \\
\hline BFI10 & 3,96 & 0,873 & $-1,046$ & 1,724 & 0,202 & 0,278 & 0,224 & 0,151 & 0,568 & 0,940 & 0,542 \\
\hline BFI12 (I) & 3,52 & 1,084 & $-0,370$ & $-0,603$ & 0,180 & $-0,226$ & 0,115 & 0,037 & 0,474 & 0,821 & 0,634 \\
\hline BFI17 & 4,05 & 0,950 & $-1,064$ & 0,998 & $-0,031$ & 0,155 & 0,193 & $-0,026$ & 0,599 & 0,946 & 0,818 \\
\hline BFI20 & 3,94 & 0,901 & $-0,853$ & 0,605 & 0,438 & 0,246 & 0,422 & 0,335 & 0,653 & 0,861 & 0,324 \\
\hline BFI23 & 3,55 & 1,008 & $-0,489$ & 0,004 & 0,282 & 0,025 & 0,261 & 0,309 & 0,740 & 0,945 & 0,623 \\
\hline BFI31 & 3,78 & 0,880 & $-0,606$ & 0,280 & 0,268 & 0,308 & 0,356 & 0,196 & 0,464 & 0,837 & 0,275 \\
\hline BFI39 & 3,52 & 1,095 & $-0,359$ & $-0,562$ & 0,067 & 0,206 & 0,008 & $-0,002$ & 0,486 & 0,949 & 0,805 \\
\hline BFI44 (I) & 3,81 & 1,104 & $-0,506$ & $-0,726$ & $-0,004$ & $-0,100$ & $-0,005$ & $-0,171$ & 0,448 & 0,777 & 0,803 \\
\hline$\Phi_{\mathrm{F}}$ & & & & & 0,907 & 0,665 & 0,886 & 0,926 & 0,890 & & \\
\hline $\mathrm{F}_{1}$ & - & - & - & - & 1 & & & & & & \\
\hline $\mathrm{F}_{2}$ & - & - & - & - & $-0,221$ & 1 & & & & & \\
\hline $\mathrm{F}_{3}$ & - & - & - & - & 0,121 & 0,006 & 1 & & & & \\
\hline $\mathrm{F}_{4}$ & - & - & - & - & $-0,311$ & $-0,170$ & $-0,560$ & 1 & & & \\
\hline $\mathrm{F}_{5}$ & - & - & - & - & $-0,163$ & 0,319 & $-0,122$ & $-0,347$ & 1 & & \\
\hline a & & & & & 0,794 & 0,621 & 0,701 & 0,742 & 0,763 & & \\
\hline $95 \%$ IC & - & - & - & - & $\begin{array}{l}{[0,751,} \\
0,831]\end{array}$ & $\begin{array}{l}{[0,550} \\
0,683]\end{array}$ & $\begin{array}{l}{[0,642} \\
0,752]\end{array}$ & $\begin{array}{l}{[0,689} \\
0,787]\end{array}$ & $\begin{array}{l}{[0,714,} \\
0,805]\end{array}$ & & \\
\hline Oordinal & - & - & - & - & 0,837 & 0,615 & 0,797 & 0,883 & 0,811 & & \\
\hline$\omega$ & - & - & - & - & 0,845 & 0,618 & 0,805 & 0,885 & 0,814 & & \\
\hline$H$ & - & - & - & - & 0,888 & 0,627 & 0,818 & 0,895 & 0,834 & & \\
\hline
\end{tabular}

Nota. $n=332$; (I): ítem invertido; $M$ : media aritmética. $D E$ : desviación estándar; $g_{1}$ : asimetría de Fisher; g $g_{2}$ curtosis de Fisher; F 1 : Extraversión; $\mathrm{F}_{2}$ : Afabilidad; $\mathrm{F}_{3}$ : Responsabilidad; $\mathrm{F}_{4}$ : Neuroticismo; F5: Apertura; $\Phi_{\mathrm{I}}$ : coeficiente de congruencia factorial del ítem; $\Phi_{\mathrm{F}}$ : coeficiente de congruencia factorial del factor; ISF: índice de simplicidad factorial; $\alpha_{\text {ordinal }}$ coeficiente alfa ordinal; $\omega$ : coeficiente omega; $H$ : coeficiente $H$. 
Tabla 3

Correlaciones entre Factores del BFI

\begin{tabular}{lccccc}
\hline & $\mathrm{F}_{1}$ & $\mathrm{~F}_{2}$ & $\mathrm{~F}_{3}$ & $\mathrm{~F}_{4}$ & $\mathrm{~F}_{5}$ \\
\hline $\mathrm{F}_{1}$ & 1 & & & & \\
$\mathrm{~F}_{2}$ & $0,398^{*}$ & 1 & & & \\
$\mathrm{~F}_{3}$ & $0,279^{*}$ & $0,170^{*}$ & 1 & & \\
$\mathrm{~F}_{4}$ & $-0,198^{*}$ & 0,104 & $-0,233^{*}$ & 1 & \\
$\mathrm{~F}_{5}$ & $0,403^{*}$ & $0,358^{*}$ & $0,414^{*}$ & $-0,200^{*}$ & 1 \\
\hline
\end{tabular}

Nota. $n=332 ;{ }^{*} p<0,01 ; \mathrm{F}_{1}$ : Extraversión; $\mathrm{F}_{2}$ : Afabilidad; $\mathrm{F}_{3}:$ Responsabilidad; $\mathrm{F}_{4}$ : Neuroticismo; F5: Apertura.

\section{Análisis de Consistencia Interna}

La confiabilidad de las puntuaciones fue adecuada para propósitos de investigación (Merino Soto et al., 2014) y los valores de los coeficientes de confiabilidad basados en variables latentes ( $\omega$, aordinal y $H$ ) fueron aceptables (Gadermann, Guhn \& Zumbo, 2012; Hancock \& Mueller, 2001), como se aprecia en la Tabla 2, lo que brinda evidencias adicionales de la consistencia del instrumento, aunque los indicadores del segundo factor (Afabilidad) no son adecuados.

\section{Discusión}

El objetivo del presente estudio fue obtener las primeras evidencias de validez y confiabilidad del BFI en Perú. En líneas generales, el objetivo fue cumplido, obteniéndose una versión inicial del BFI que puede ser usada para fines de investigación y, más adelante, implementar estudios para incrementar su capacidad predictiva. En relación con la replicabilidad del modelo, un resultado que puede destacarse es que los factores Extraversión, Responsabilidad y Neuroticismo parecen robustos y, contrariamente a lo que señala la literatura (e.g., Fetvadjiev \& van de Vijver, 2015), el factor Apertura también. Este factor causa gran controversia. No obstante, el factor Afabilidad mostró un comportamiento poco estable, lo que coincide con un estudio realizado en México (Reyes Zamorano et al., 2014).

Respecto de la estructura factorial del BFI, el análisis desarrollado a través de la rotación procrusteana replica de forma aceptable la configuración original del instrumento, salvo por la eliminación de siete ítems, de los cuales cinco se califican en escala invertida (ítem 2: "Tiende a ser criticón"; ítem 13: "Inicia disputas con los demás"; ítem 22: "Es a veces maleducado con los demás"; ítem 33: "Es a veces frío y distante" y el ítem 8: "A veces puede ser algo descuidado") y todos presentaron elevada complejidad factorial con los factores Extraversión y Neuroticismo. No es sorpresivo que cinco de los estos siete ítems pertenezcan originalmente al factor Afabilidad, ya que en otros reportes se muestran correlaciones no despreciables con Extraversión y Neuroticismo (Aluja et al., 2005; Benet-Martínez \& John, 1998; Denissen et al., 2008; García et al., 2012; Gurven et al., 2013; McCrae et al., 1996). Asimismo, en otro estudio en español con el BFI los ítems eliminados aquí muestran el mismo comportamiento poco consistente y con débil discriminación (Reyes Zamorano et al., 2014). Quizá esto sea porque tanto Extraversión como Afabilidad son sensibles a las diferencias culturales y no son siempre diferenciados (Gurven et al., 2013) o porque simplemente los ítems eliminados no tienen relevancia local para el constructo. Entonces, es probable que estos sean relevantes para la evaluación de la Afabilidad en otros contextos culturales (e.g., anglosajones), pero su significado adopta una forma menos diferenciada de otros factores en los estudios en español. Es lícito suponer esto, pues la interacción con las personas (Extraversión) muchas veces está cargada de contenido emocional (Neuroticismo), lo que podría afectar la forma de relacionarse de los individuos (Afabilidad).

Por otro lado, el ítem eliminado de Responsabilidad (“A veces puede ser algo descuidado”) quizá haya sido respondido con elevada deseabilidad social (los encuestados fueron universitarios). Finalmente, la eliminación de un ítem de Apertura ("Le gusta reflexionar, jugar con las ideas") indicaría que la actividad reflexiva no se asocia claramente con el constructo que se pretende evaluar. No obstante, fue permitido cierto nivel de complejidad factorial entre los 37 ítems restantes con el objetivo de obtener una versión del BFI que maximice la discriminación, pero que no vaya en contra de los postulados originales del modelo de los $5 \mathrm{GF}$. 
Las correlaciones bivariadas observadas corroboran las hipótesis de estudio, dado que Extraversión se relaciona directamente con Afabilidad y Apertura del mismo modo que en otros estudios (Benet-Martínez \& John, 1998; Denissen et al., 2008; García et al., 2012; Gurven et al., 2013), así como con Responsabilidad (Denissen et al., 2008; Gurven et al., 2013), aunque esta última asociación no ha recibido tanto respaldo como las primeras. Por otro lado, la relación inversa de Neuroticismo con Extraversión y Responsabilidad ha sido ampliamente documentada por la literatura (Benet-Martínez \& John, 1998; Denissen et al., 2008; García et al., 2012; Gurven et al., 2013), aunque no se asoció con Afabilidad, a diferencia de otros estudios donde sí se evidenció un patrón correlacional inverso (Benet-Martínez \& John, 1998; Denissen et al., 2008; Gurven et al., 2013). Por último, si bien no se especificó como hipótesis de investigación la asociación entre Apertura con Afabilidad y Responsabilidad, debido a que solo una investigación da cuenta de esas relaciones (Gurven et al., 2013), en el presente estudio sí se asociaron. En resumen, las correlaciones observadas brindan evidencia favorable de validez con relación a otras variables (American Educational Research Association [AEA], American Psychological Association [APA] \& National Council on Measurement in Education [NCME], 2014) de los factores de la versión de 37 ítems del BFI, dada su compatibilidad con estudios previos realizados en diversos contextos culturales y con muestras heterogéneas. Es necesario mencionar que, en el marco de las rotaciones procrusteanas, la congruencia entre la matriz observada y la TM dependen de varias características del modelo planteado, incluyendo la cantidad de ítems y las especificaciones en la TM, así como el número de cargas salientes por factor (Paunonen, 1997), por lo que es esperable que esta situación afecte a las correlaciones interfactoriales, dadas las particularidades de la rotación procrusteana oblicua (Gruvaeus, 1970). Esto explicaría las diferencias observadas con relación a la matriz de correlaciones basadas en puntuaciones observadas.

Sobre la confiabilidad, cabe resaltar que las condiciones para aplicar el coeficiente alfa de Cronbach no fueron cumplidas, ya que algunos ítems presentaron coeficientes de configuración de mayor magnitud que otros y en unos casos la diferencia fue notoria. Por ello, fueron consideradas otras alternativas de análisis (ardinal, $\omega$ y $H$ ), cuyas magnitudes fueron superiores. Estos resultados se deben a que los coeficientes basados en variables latentes no requieren la tau-equivalencia como condición necesaria para su cálculo, ya que es suficiente con que se traten de medidas congenéricas (Dunn, Baguley \& Brunsden, 2014).

Algunas limitaciones surgieron en el proceso de investigación. En primer lugar, el carácter no probabilístico de la muestra, al ser facilitada por el acceso de los investigadores al grupo, no permite hacer generalizaciones hacia otras poblaciones de universitarios, pero, considerando el tamaño muestral moderado y los resultados favorables respecto de la estructura del instrumento y de su confiabilidad, es plausible considerarlo como un instrumento robusto. Sin embargo, dada la homogeneidad del grupo respecto de su origen, la capacidad para generalizar a otros grupos con similares características es considerada una ventaja, más aún si el objetivo psicométrico del estudio fue evaluar inicialmente el BFI en Perú. En segundo lugar, el desbalance entre varones y mujeres pudo haber influido en los resultados, aunque, con base en la evidencia previa que indica la invarianza entre sexos (Chiorri, Marsh, Ubbiali \& Donati, 2016; Kokkinos \& Markos, 2017), no sería un problema el análisis conjunto de ambos grupos. Sin embargo, la corroboración de este supuesto es tarea pendiente para próximas investigaciones, así como el incremento de la muestra de estudio y el tipo de muestreo (i.e., probabilístico), para llegar a conclusiones más confiables acerca de la invarianza factorial. En tercer lugar, la ausencia de criterios externos debe ser abordada en futuros estudios, mediante correlatos teóricamente fundamentados. En cuarto lugar, aunque se esperaría una elevada estabilidad de las respuestas, dado que la personalidad es una característica estable, se requieren estimaciones de confiabilidad test-retest. En quinto lugar, la ausencia del heterorreporte no permite conocer un criterio de validación clave de medidas como esta. Finalmente, el análisis debe extenderse a grupos más heterogéneos en cuanto a actividad (e.g., trabajadores) y edad (e.g., adolescentes), ya que existe evidencia de que los 5GF tienen un comportamiento distinto según el grupo etario (Soto et al., 2008; Soto, John, Gosling \& Potter, 2011), grado de instrucción (Rammstedt, Kemper, Klein, Beierlein \& Kovaleva, 2013) e inclusive procedencia cultural (Fetvadjiev \& van der Vijver, 2015; Gurven et al., 2013; Rammstedt, Kemper, Klein et al., 2013).

Por otro lado, sería necesario realizar estudios adicionales enfocados en el proceso de respuesta (AEA, APA \& NCME, 2014), utilizando entrevistas cognitivas. Esto brindaría información sobre la posible causa de la eliminación de los ítems y ayudaría a fijar directrices para modificar o crear nuevos ítems.

Cabe resaltar la utilidad del procedimiento analítico implementado: rotación procrusteana. Este es de uso poco frecuente, ya que generalmente las opciones para la validación de la estructura interna del instrumento han sido el AFE y AFC (Ferrando \& Anguiano-Carrasco, 2010; Lloret-Segura et al., 2014). Este procedimiento se encuentra en un punto entre ambas tradiciones factoriales y puede ser útil en casos como 
este (Ferrando \& Lorenzo-Seva, 2000, 2014), en los que las características de los constructos a evaluar no son apropiadamente analizadas por un enfoque liberal o restrictivo.

El presente estudio concluye que el BFI muestra propiedades psicométricas satisfactorias, evidenciando una estructura factorial coherente, relaciones aceptables entre dimensiones e indicadores de confiabilidad satisfactorios, tanto con puntuaciones observadas como con variables latentes. No obstante, estos hallazgos deben ser replicados en muestras con distintas características (e.g., población general) e incorporando otras estrategias de validación, sobre todo su relación con otros constructos.

\section{Referencias}

Aluja, A. \& Blanch, Á. (2003). Replicabilidad de los factores de segundo orden del 16PF-5 en muestras americanas y españolas. Psicothema, 15, 309-314. Extraído de www.psicothema.com/pdf/1062.pdf

Aluja, A., García, Ó., García, L. F. \& Seisdedos, N. (2005). Invariance of the "NEO-PI-R" factor structure across exploratory and confirmatory factor analyses. Personality and Individual Differences, 38, 1879-1889. https://doi.org/10.1016/j.paid.2004.11.014

American Educational Research Association, American Psychological Association \& National Council on Measurement in Education (2014). Standards for educational and psychological testing ( $8^{\mathrm{a}}$ ed.). Washington, DC: American Educational Research Association.

Benet-Martínez, V. \& John, O. P. (1998). Los cinco grandes across cultures and ethnic groups: Multitrait-multimethod analyses of the big five in Spanish and English. Journal of Personality and Social Psychology, 75, 729-750. https://doi.org/10.1037//00223514.75.3.729

Browne, M. W. (1972a). Orthogonal rotation to a partially specified target. British Journal of Mathematical and Statistical Psychology, 25, 115-120. https://doi.org/10.1111/j.2044-8317.1972.tb00482.x

Browne, M. W. (1972b). Oblique rotation to a partially specified target. British Journal of Mathematical and Statistical Psychology, 25, 207-212. https://doi.org/10.1111/j.2044-8317.1972.tb00492.x

Carciofo, R., Yang, J., Song, N., Du, F. \& Zhang, K. (2016). Psychometric evaluation of Chinese-language 44-item and 10-item Big Five Personality Inventories, including correlations with chronotype, mindfulness and mind wandering. PLOS ONE, 11(2), e0149963. https://doi.org/10.1371/journal.pone.0149963

Castro Solano, A. \& Casullo, M. M. (2001). Rasgos de personalidad, bienestar psicológico y rendimiento académico en adolescentes argentinos. Interdisciplinaria, 18, 65-85. Extraído de http://www.redalyc.org/articulo.oa?id=18011326003

Chiorri, C., Marsh, H. W., Ubbiali, A. \& Donati, D. (2016). Testing the factor structure and measurement invariance across gender of the Big Five Inventory through exploratory structural equation modeling. Journal of Personality Assessment, 98, 88-99. https://doi.org/10.1080/00223891.2015.1035381

Cliff, N. (1966). Orthogonal rotation to congruence. Psychometrika, 31, 33-42. https://doi.org/10.1007/bf02289455

Costa, P. T. \& McCrae, R. R. (1992). Revised NEO Personality Inventory (NEO PI-R) and NEO Five-Factor Inventory (NEO - FFI) manual. Odessa, FL: Psychological Assessment Resources.

Denissen, J. J. A., Geenen, R., van Aken, M. A. G., Gosling, S. D. \& Potter, J. (2008). Development and validation of a Dutch translation of the Big Five Inventory (BFI). Journal of Personality Assessment, 90, 152-157. https://doi.org/10.1037/t62652-000

Digman, J. M. (1967). The Procrustes class of factor-analytic transformations. Mutivariate Behavioral Research, $2,89-94$. https://doi.org/10.1080/00273176709595116

Dominguez, S. (2012). Propuesta para el cálculo del alfa ordinal y theta de Armor. Revista de Investigación en Psicología, 15 (1), $213-217$. https://doi.org/10.15381/rinvp.v15i1.3684

Dominguez Lara, S. (2014). ¿Matrices policóricas/tetracóricas o matrices Pearson? Un estudio metodológico. Revista Argentina de Ciencias del Comportamiento, 6(1), 39-48. Extraído de https://revistas.unc.edu.ar/index.php/racc/article/view/6357/Lara2014

Dominguez-Lara, S. (2016). Evaluación de la confiabilidad del constructo mediante el coeficiente H: breve revisión conceptual y aplicaciones. Psychologia, 10(2), 87-94. https://doi.org/10.21500/19002386.2134

Dominguez-Lara, S. \& Merino-Soto, C. (2015). ¿Por qué es importante reportar los intervalos de confianza del coeficiente alfa de Cronbach? Revista Latinoamericana de Ciencias Sociales, Niñez y Juventud, 13, 1326-1328. Extraído de http://revistaumanizales.cinde.org.co/rlcsnj/index.php/Revista-Latinoamericana/article/view/2030/629

Dunn, T. J., Baguley, T. \& Brunsden, V. (2014). From alpha to omega: A practical solution to the pervasive problem of internal consistency estimation. British Journal of Psychology, 105, 399-412. https://doi.org/10.1111/bjop.12046

Elosua Oliden, P. \& Zumbo, B. D. (2008). Coeficientes de fiabilidad para escalas de respuesta categórica ordenada. Psicothema, 20, 896901. Extraído de http://www.psicothema.com/pdf/3572.pdf

Ferguson, C. J. (2009). An effect size primer: A guide for clinicians and researchers. Professional Psychology: Research and Practice, 40, 532-538. https://doi.org/10.1037/a0015808

Ferrando, P. J. \& Anguiano-Carrasco, C. (2010). El análisis factorial como técnica de investigación en psicología. Papeles del Psicólogo, 31, 18-33. Extraído de www.papelesdelpsicologo.es/pdf/1793.pdf

Ferrando, P. J. \& Lorenzo-Seva, U. (2000). Unrestricted versus restricted factor analysis of multidimensional test items: Some aspects of the problem and some suggestions. Psicológica, 21, 301-323. Extraído de https://www.uv.es/revispsi/articulos3.00/ferran7

Ferrando, P. J. \& Lorenzo-Seva, U. (2014). El análisis factorial exploratorio de los ítems: algunas consideraciones adicionales. Anales de Psicología, 30, 1170-1175. https://doi.org/10.6018/analesps.30.3.199991

Fetvadjiev, V. \& van der Vijver, F. J. R. (2015). Universality of the five-factor model of personality. En J. D. Wright (Ed.), International encyclopedia of the social \& behavioral sciences ( $2^{\mathrm{a}}$ ed., Vol. 9, pp. 249-253). London, Reino Unido: Elsevier. https://doi.org/10.1016/b9780-08-097086-8.25067-8

Fisher, R. A. (1950). Statistical methods for research workers (11 $1^{\mathrm{a}}$ ed. rev.). Edinburgh, Reino Unido: Oliver \& Boyd.

Fleming, J. S. \& Merino Soto, C. (2005). Medidas de simplicidad y ajuste factorial: un enfoque para la evaluación de escalas construidas factorialmente. Revista de Psicología-PUCP, 23, 249-266. Extraído de http://revistas.pucp.edu.pe/index.php/psicologia/article/view/2150/2083 
Fossati, A., Borroni, S., Marchione, D. \& Maffei, C. (2011). The Big Five Inventory (BFI): Reliability and validity of its Italian translation in three independent nonclinical samples. European Journal of Psychological Assessment, 27, 50-58. https://doi.org/10.1027/1015$5759 / \mathrm{a} 000043$

Gadermann, A. M., Guhn, M. \& Zumbo, B. D. (2012). Estimating ordinal reliability for Likert-type and ordinal item response data: A conceptual, empirical, and practical guide. Practical Assessment, Research \& Evaluation, 17, artículo 3. Extraído de http://pareonline.net/getvn.asp? $=17 \& n=3$

García, L. F., Escorial, S., García, Ó., Blanch, A. \& Aluja, A. (2012). Structural analysis of the facets and domains of the ZuckermanKuhlman-Aluja Personality Questionnaire (ZKA-PQ) and the NEO PI-R. Journal of Personality Assessment, 94, 156-163. https://doi.org/10.1080/00223891.2011.645935

Goldberg, L. R. (1990). An alternative "description of personality": The big-five factor structure. Journal of Personality and Social Psychology, 59, 1216-1229. https://doi.org/10.1037//0022-3514.59.6.1216

Goldberg, L. R., Johnson, J. A., Eber, H. W., Hogan, R., Ashton, M. C., Cloninger, C. R. \& Gough, H. G. (2006). The international personality item pool and the future of public-domain personality measures. Journal of Research in Personality, 40, 84-96. https://doi.org/10.1016/j.jrp.2005.08.007

Gruvaeus, G. T. (1970). A general approach to Procrustes pattern rotation. Psychometrika, 35, 493-505. https://doi.org/10.1007/BF02291822

Gurven, M., von Rueden, C., Massenkoff, M., Kaplan, H. \& Lero Vie, M. (2013). How universal is the big five? Testing the five-factor model of personality variation among forager-farmers in the Bolivian Amazon. Journal of Personality and Social Psychology, 104, 354-370. https://doi.org/10.1007/bf02291822

Hancock, G. R. \& Mueller, R. O. (2001). Rethinking construct reliability within latent variable systems. En R. Cudeck, S. H. C. du Toit \& D. Sörbom (Eds.), Structural equation modeling: Past and present. A Festschrift in honor of Karl G. Jöreskog (pp. 195-261). Chicago, IL: Scientific Software International.

Henrich, J., Heine, S. J. \& Norenzayan, A. (2010). The weirdest people in the world? Behavioral and Brain Sciences, 33, 61-83. https://doi.org/10.1017/s0140525x0999152x

Horn, J. L. (1965). A rationale and test for the number of factors in factor analysis. Psychometrika, 30, 179-185. https://doi.org/10.1007/bf02289447

Hu, L. -t. \& Bentler, P. M. (1998). Fit indices in covariance structure modeling: Sensitivity to underparameterized model misspecification. Psychological Methods, 3, 424-453. https://doi.org/10.1037//1082-989x.3.4.424

John, O. P., Donahue, E. M. \& Kentle, R. L. (1991). The Big Five Inventory - versions 4a and 54. Berkeley, CA: Institute of Personality and Social Research.

Kaiser, H. F. (1974). An index of factorial simplicity. Psychometrika, 39, 31-36. https://doi.org/10.1007/bf02291575

Kokkinos, C. M. \& Markos, A. (2017). The Big Five Questionnaire for Children (BFQ-C): Factorial invariance across sex and age in a Greek sample of preadolescents. European Journal of Psychological Assessment, 33, 129-133. https://doi.org/10.1027/10155759/a000273

Krueger, R. F., Eaton, N. R., Clark, L. A., Watson, D., Markon, K. E., Derringer, J. ... Livesley, W. J. (2011). Deriving an empirical structure of personality pathology for DSM-5. Journal of Personality Disorders, 25, 170-191. https://doi.org/10.1521/pedi.2011.25.2.170

Leung, D. Y. P., Wong, E. M. L., Chan, S. S. C. \& Lam, T. H. (2013). Psychometric properties of the Big Five Inventory in a Chinese sample of smokers receiving cessation treatment: A validation study. Journal of Nursing Education and Practice, 3(6), 1-10. https://doi.org/10.5430/jnep.v3n6p1

Lloret-Segura, S., Ferreres-Traver, A., Hernández-Baeza, A. \& Tomás-Marco, I. (2014). El análisis factorial exploratorio de los ítems: una guía práctica, revisada y actualizada. Anales de Psicología, 30, 1151-1169. https://doi.org/10.6018/analesps.30.3.199361

Lorenzo-Seva, U. \& Ferrando, P. J. (2013). FACTOR 9.2: A comprehensive program for fitting exploratory and semiconfirmatory factor analysis and IRT models. Applied Psychological Measurement, 37, 497-498. https://doi.org/10.1177/0146621613487794

Lorenzo-Seva, U. \& ten Berge, J. M. F. (2006). Tucker's congruence coefficient as a meaningful index of factor similarity. Methodology, 2, 57-64. https://doi.org/10.1027/1614-2241.2.2.57

Mardia, K. V. (1970). Measures of multivariate skewness and kurtosis with applications. Biometrika, 57, 519-530. https://doi.org/10.2307/2334770

McCrae, R. R. \& Terracciano, A. (2005). Universal features of personality traits from the observer's perspective: Data from 50 cultures. Journal of Personality and Social Psychology, 88, 547-561. https://doi.org/10.1037/0022-3514.88.3.547

McCrae, R. R., Zonderman, A. B., Costa Jr., P. T., Bond, M. H. \& Paunonen, S. V. (1996). Evaluating replicability of factors in the Revised NEO Personality Inventory: Confirmatory factor analysis versus Procrustes rotation. Journal of Personality and Social Psychology, 70, 552-566. https://doi.org/10.1037/0022-3514.70.3.552

McDonald, R. P. (1999). Test theory: A unified treatment. Mahwah, NJ: Lawrence Erlbaum.

Merino, C. \& Grimaldo Muchotrigo, M. P. (2011). Complejidad factorial y conductas moralmente controversiales. Revista Argentina de Ciencias del Comportamiento, 3(3), 38-43. Extraído de https://revistas.unc.edu.ar/index.php/racc/article/download/5198/5363

Merino Soto, C., Navarro Loli, J. \& García Ramirez, W. (2014). Revisión de la consistencia interna del Inventario de Inteligencia Emocional de Bar-On, EQ-I: YV. Revista Peruana de Psicología y Trabajo Social, 3(1),141-154. Extraído de http://revistas.uigv.edu.pe/index.php/psicologia/article/view/61/69

Ortet, G., Ibáñez, M. I., Ruipérez, M. A., Villa, H., Moya, J. \& Escrivá, P. (2007). Adaptación para adolescentes de la versión española del NEO PI-R (JS NEO). Psicothema, 19, 263-268. Extraído de http://www.psicothema.com/pdf/3358.pdf

Paunonen, S. V. (1997). On chance and factor congruence following orthogonal Procrustes rotation. Educational and Psychological Measurement, 57, 33-59. https://doi.org/10.1177/0013164497057001003

Rammstedt, B. \& John, O. P. (2007). Measuring personality in one minute or less: A 10-item short version of the Big Five Inventory in English and German. Journal of Research in Personality, 41, 203-212. https://doi.org/10.1016/j.jrp.2006.02.001

Rammstedt, B., Kemper, C. J. \& Borg, I. (2013). Correcting big five personality measurements for acquiescence: An 18-country crosscultural study. European Journal of Personality, 27, 71-81. https://doi.org/10.1002/per.1894

Rammstedt, B., Kemper, C. J., Klein, M. C., Beierlein, C. \& Kovaleva, A. (2013). Eine kurze Skala zur Messung der fünf Dimensionen der Persönlichkeit [Una escala corta para medir las cinco dimensiones de la personalidad]. Methoden, Daten, Analysen, 7, 233-249. Extraído de https://www.ssoar.info/ssoar/bitstream/handle/document/31213/ssoar-2012-rammstedt_et_al-eine_kurze_skala_zur_messung.pdf?sequence=1

Reyes Zamorano, E., Álvarez Carrillo, C., Peredo Silva, A., Miranda Sandoval, A. \& Rebolledo Pastrana, I. M. (2014). Psychometric properties of the Big Five Inventory in a Mexican sample. Salud Mental, 37, 491-497. https://doi.org/10.17711/sm.01853325.2014 .059 
Rodríguez Ayán, M. N. \& Ruiz Díaz, M. A. (2008). Atenuación de la asimetría y de la curtosis de las puntuaciones observadas mediante transformaciones de variables: incidencia sobre la estructura factorial. Psicológica, 29, 205-227. Extraído de https://www.uv.es/revispsi/articulos2.08/6RODRIGUEZ.pdf

Romano, J. L., Kromrey, J. D. \& Hibbard, S. T. (2010). A Monte Carlo study of eight confidence interval methods for coefficient alpha. Educational and Psychological Measurement, 70, 376-393. https://doi.org/10.1177/0013164409355690

Romano, J. L., Kromrey, J. D., Owens, C. M. \& Scott, H. M. (2011). Confidence interval methods for coefficient alpha on the basis of discrete, ordinal response items: Which one, if any, is the best? The Journal of Experimental Education, 79, 382-403. https://doi.org/10.1080/00220973.2010.510859

Schmitt, D. P., Allik, J., McCrae, R. R. \& Benet-Martínez, V. (2007). The geographic distribution of big five personality traits: Patterns and profiles of human self-description across 56 nations. Journal of Cross-Cultural Psychology, 38, 173-212. https://doi.org/10.1177/0022022106297299

Schönemann, P. H. (1966). A generalized solution of the orthogonal Prcrustes problem. Psychometrika, 31, 1-10. https://doi.org/10.1007/bf02289451

Soto, C. J. \& John, O. P. (2009). Ten facets scales for the Big Five Inventory: Convergence with NEO PI-R facets, self-peer agreement, and discriminant validity. Journal of Research in Personality, 43, 84-90. https://doi.org/10.1016/j.jrp.2008.10.002

Soto, C. J., John, O. P., Gosling, S. D. \& Potter, J. (2008). The developmental psychometrics of big five self-reports: Acquiescence, factor structure, coherence, and differentiation from ages 10 to 20. Journal of Personality and Social Psychology, 94, 718-737. https://doi.org/10.1037/e511092014-204

Soto, C. J., John, O. P., Gosling, S. D. \& Potter, J. (2011). Age differences in personality traits from 10 to 65 : Big five domains and facets in a large cross-sectional sample. Journal of Personality and Social Psychology, 100, 330-348. https://doi.org/10.1037/a0021717

ten Berge, J. M. F. (1986). Some relationships between descriptive comparisons of components from different studies. Mutivariate Behavioral Research, 21, 29-40. https://doi.org/10.1207/s15327906mbr2101_2

Tucker, L. R. (1951). A method for synthesis of factor analysis studies (Personnel Research Section Report N 984). Washington, DC: Department of the Army.

Ubbiali, A., Chiorri, C., Hampton, P. \& Donati, D. (2013). Psychometric properties of the Italian adaptation of the Big Five Inventory (BFI). Bollettino di Psicologia Applicata, 266, 37-46. Extraído de https://www.researchgate.net/publication/250662562_Psychometric_properties_of_the_Italian_adaptation_of_the_Big_Five_Inventory_BFI)

Worrell, F. C. \& Cross Jr., W. E. (2004). The reliability and validity of Big Five Inventory scores with African American college students. Journal of Multicultural Counseling and Development, 32, 18-32. https://doi.org/10.1002/j.2161-1912.2004.tb00358.x

Yamagata, S., Suzuki, A., Ando, J., Ono, Y., Kijima, N., Yoshimura, K. ... Jang, K. L. (2006). Is the genetic structure of human personality universal? A cross-cultural twin study from North America, Europe, and Asia. Journal of Personality and Social Psychology, 90, 987-998. https://doi.org/10.1037/0022-3514.90.6.987

Yang, J. -F. (2010). Cross-cultural personality assessment: The Revised NEO Personality Inventory in China. Social Behavior and Personality, 38, 1097-1104. https://doi.org/10.2224/sbp.2010.38.8.1097

Fecha de recepción: Junio de 2016.

Fecha de aceptación: Enero de 2018. 